cutaneous and cerebral vasculitis. The levels of FA and N-1 also correlated with more pronounced radiological changes (X-ray stage III). FA circulating inhibitor of ectopic calcification. $\mathrm{N}-1$ level is positively correlated with systolic blood pressure.

Conclusion: A low level of $A$ and FA, a high level of $V$ and $N-1$ is characteristic of RA with the presence of high activity and positivity in the RF and Anti-CCP. An increased level of B is determined by more than $90 \%$ of patients, which indicates its high pro-inflammatory activity. The level of $\mathrm{F}$ and $\mathrm{N}-1$ is also associated with the degree of damage to bone tissue (stage III, a lot of erosion). A positive correlation of level $\mathrm{V}$ and $\mathrm{N}-1$, negative $\mathrm{A}$ and $\mathrm{FA}$ with the severity of inflammation in RA confirms the involvement of these proteins in the pathogenesis. A high level of $A$ and $V$ increases the risk of developing cardiovascular diseases and their complications, the effect of $\mathrm{N}-1$ and FA is being studied. The effect of cytokines on osteoclasts and osteoblasts in RA is ambiguous

References:

[1] Visfatin and Rheumatoid Arthritis: Pathogenetic Implications and Clinical Utility. Polyakova Y. Curr Rheumatol Rev.2019

[2] Serum nesfatin -1 as a marker of systemic inflammation in rheumatoid arthritis. Kvlividze T. Klinicheskaya Laboratornaya Diagnostika.2019; 64 (1):53-56 (in Russ)

[3] Fetuin-A. Novel hepatokine in rheumatoid arthritis laboratory diagnostics. Papichev E. Klinicheskaya Laboratornaya Diagnostika.2018; 63 (12):756760 (in Russ)

Disclosure of Interests: None declared

DOI: 10.1136/annrheumdis-2020-eular.1226

\section{AB0104 CORRELATION OF PLASMA 14-3-3H LEVELS WITH DISEASE ACTIVITY MEASURES IN METHOTREXATE- NAÏVE RA PATIENTS TREATED WITH UPADACITINIB MONOTHERAPY IN THE SELECT-EARLY PHASE 3 STUDY}

T. Sornasse $^{1}$, S. Chahal ${ }^{2}$, Y. Gui ${ }^{2}$, N. Nagarajan ${ }^{2}$, A. Friedman ${ }^{3}$, N. Biln². ${ }^{1}$ AbbVie Immunology Clinical Development, Redwood City, United States of America; ${ }^{2}$ Augurex, Vancouver, Canada; ${ }^{3}$ AbbVie Immunology Clinical Development, North Chicago, United States of America

Background: Early diagnosis and treat-to-target strategies in RA improve clinical and radiographic outcomes. $14-3-3 \eta$ is a soluble diagnostic biomarker that is involved in the pathogenesis of RA (1) including the potent activation of key signalling cascades such as the JAK-STAT pathway and whose initial expression coincides with a transition to synovitis. In undifferentiated arthralgia, 14-3-3n independently predicts the development of RA. In confirmed RA, 14-3-3n levels decrease with treatment response (2) and those changes are associated with clinical and radiographic outcomes, including the prediction of joint damage progression in patients who have achieved clinical remission. Upadacitinib (UPA), an oral JAK1-selective inhibitor, demonstrated significant and clinically meaningful improvements in RA vs. methotrexate (MTX) in the SELECT-EARLY phase 3 study (3).

Objectives: To determine the impact of treatment with UPA monotherapy $15 \mathrm{mg}$ QD on the levels of plasma 14-3-3n and to explore its relationship with clinical measures in early MTX-naïve RA patients.

Methods: Patients from the SELECT-EARLY study were randomly selected (UPA $n=100 ;$ MTX $n=100$ ) from the pool of patients with available plasma samples. 14-3-3n tests were performed at Augurex according to standard operating procedures. Concentrations $(\mathrm{ng} / \mathrm{mL})$ were log-transformed for analysis. Non-parametric correlations between biomarker data and clinical end points were derived using the Spearman method. Changes in 14-3-3n over time were compared using a Repeated Measure Mixed Linear Model. All statistical analyses were conducted in JMP 14.1 (SAS Institute).

Results: At baseline, $79 \%$ of patients were $14-3-3 \eta$ positive ( $\geq 0.19 \mathrm{ng} / \mathrm{mL}$ ) with similar levels in both arms. Baseline levels of $14-3-3 n$ correlated significantly with baseline disease activity measures (CDAI $\rho=0.164, p=0.042$; DASCRP $\rho=0.222, p=0.004$; and SDAI $\rho=0.177, p=0.028$ ) but not with baseline $\operatorname{mTSS}(\rho=-0.021, p=0.787)$; of note baseline mTSS were relatively low in this subset of early RA patients (median $=2$, IQR $[0-9.5]$ ). Over time, 14-3-3n levels tended to be lower in both the UPA and MTX groups. However, only treatment with UPA for 24 weeks resulted in a significant decrease in circulating 14-3-3n ( $p=0.0002)$ (Figure 1). In addition, at week 24 in the UPA arm, changes in 14-3-3n levels correlated significantly with changes in concurrent disease activity measures $(\Delta$ CDAI $\rho=0.264, p=0.030 ; \Delta$ DASCRP $\rho=0.267, p=0.021 ;$ and $\Delta$ SDAI $\rho=0.267, p=0.028)$ but not with change in mTSS $(\rho=-0.186, p=0.111)$. In contrast to UPA, the relatively small changes in 14-3-3n induced by MTX did not correlate with any clinical measures.
Figure 1

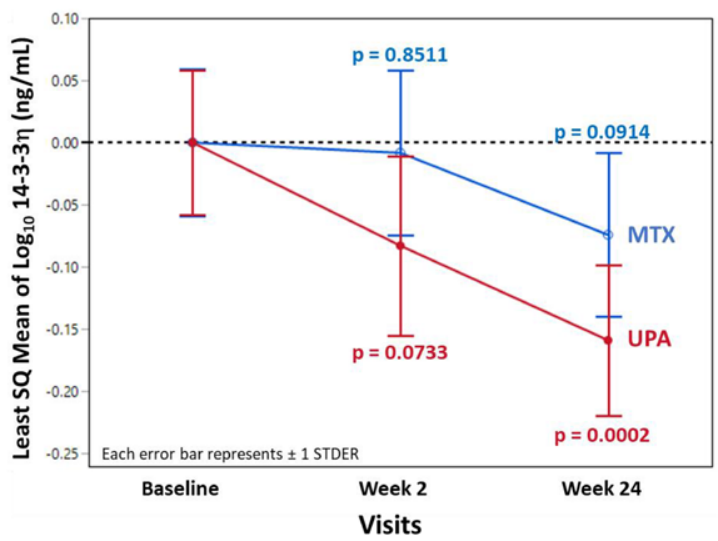

Conclusion: This study demonstrates that treatment with UPA $15 \mathrm{mg}$ QD monotherapy for 24 weeks significantly reduces the levels of circulating 14-3-3n in MTX-naïve RA patients and that these changes correlate with clinical measures of disease activity. Although we were not able to detect a clear relationship between changes in 14-3-3n and rate of structural damage progression, we would like to hypothesize that the superior clinical activity of UPA over MTX on joint damage may be related to the significant reduction in 14-3-3n induced by UPA; this hypothesis should be tested in a larger RA cohort with a larger proportion of joint damage progressors.

References:

[1] Maksymowych WP et al. Arthritis Res Ther. 2014;16(2):R99.

[2] Hirata S et al. Arthritis Res Ther. 2015;17(1):280.

[3] van Vollenhoven R et al. Arthritis Rheumatol.;2018 (Suppl 10; vol.70).

Disclosure of Interests: Thierry Sornasse Shareholder of: AbbVie Inc, Employee of: AbbVie Inc, Simran Chahal Shareholder of: Augurex Life Science Corp, Employee of: Augurex Life Science Corp, Yuan Gui Shareholder of: Augurex Life Science Corp, Employee of: Augurex Life Science Corp, Neeraja Nagarajan Employee of: Augurex Life Science Corp, Alan Friedman Shareholder of: AbbVie Inc, Employee of: AbbVie Inc, Norma Biln Shareholder of: Augurex Life Science Corp, AbbVie Inc, Employee of: Augurex Life Science Corp

DOI: 10.1136/annrheumdis-2020-eular.3239

\section{AB0105 1 CHECKPOINT MOLECULES AND PREGNANCY: ENHANCED SPD-L1 PREDICTS FLARE IN RA PREGNANCY AND POSTPARTUM}

A. L. Stefanski ${ }^{1,2}$, K. Eriksson ${ }^{1}$, A. Zbinden ${ }^{1}$, P. Villiger ${ }^{1}$, F. Förger ${ }^{1} .{ }^{1}$ Inse/spital Bern, Clinic for Rheumatology, Immunology and Allergology, Bern, Switzerland; ${ }^{2}$ Charite University Medicine, Clinic for Rheumatology, Berlin, Germany

Background: Rheumatoid arthritis (RA) is a female-predominant autoimmune disease that may affect women in childbearing age, making family planning an important issue for their life. There is a need for better understanding the mechanisms modulating RA in pregnancy and develop prognostic marker regarding adverse pregnancy outcome such as reduced birth weight and preterm delivery. As a crucial organ for peripheral tolerance during pregnancy, the placenta is expressing constitutively programmed cell death ligand 1 (PD-L1), major ligand of the inhibitory receptor PD-1 (Zhang et al, 2015). We hypothesize that the PD-1 pathway plays a central inhibitory role in regulating the course of the disease and pregnancy outcome in RA.

Objectives: To investigate the relationship between $\mathrm{PD}-1$ pathway, disease activity during pregnancy/postpartum and pregnancy outcome in RA.

Methods: We measured soluble PD-1 and PD-L1 levels by ELISA in serum samples of 27 pregnant RA patients and 25 healthy pregnant controls at different time points during pregnancy and postpartum. As for pregnancy controls, we analyzed serum samples from 28 non-pregnant RA patients and 18 non-pregnant healthy controls. The data was analyzed in correlation with disease activity (measured by DAS28-CRP) and pregnancy outcome (defined as preterm delivery and birth weight). Statistics were calculated by Mann-Whitney $U$ test and Wilcoxon test, correlations by Spearman rank test.

Results: In healthy pregnancy, SPD-L1 increases significantly in the $1^{\text {st }}$ trimeste ( $p=0,0198)$ and decreases significantly postpartum $(p=0,0029)$. sPD-L1 values are higher in non-pregnant RA patients compared to non-pregnant healthy controls $(p=0,047)$ and there are no significant changes during RA pregnancy. Postpartum SPD-L1 values are significantly higher in RA patients compared to healthy controls ( $p=0,0014)$, Fig. 1 . Notably, regarding disease activity, we noticed a significant positive correlation between the overall SPD-L1 values in 